\title{
Ant Colony Optimization based Delay and Energy Conscious Routing Protocol for Mobile Adhoc Networks
}

\author{
Arafat S.M. Qaed \\ Doctoral Scholar \\ Department of Computer Applications \\ School of Computer Science and Engineering \\ Bharathiar University \\ Coimbatore - 641046 , India
}

\author{
T. Devi \\ Reader \& HOD \\ Department of Computer Applications \\ School of Computer Science and Engineering \\ Bharathiar University \\ Coimbatore - 641 046, India
}

\begin{abstract}
Optimizing routing in mobile adhoc networks is a challenging task. Since mobile adhoc networks are highly dynamic topology natured, several routing protocols face the challenge of delay and energy conscious routing. This paper proposes a novel delay and energy conscious routing approach based on ant colony optimization. Based on the estimated delay and residual energy of the nearby nodes, "Delay and Energy Conscious Routing Protocol" (DECRP) finds the efficient node and sends the data packets through that node. Simulation results proved that DECRP reduces delay and energy consumption and increases packet delivery ratio than that of the "Adhoc On Demand Distance Vector" (AODV) protocol.
\end{abstract}

\section{KEY WORDS:}

Mobile AdHoc Networks, Delay, Energy, Routing, Ant Colony Optimization.

\section{INTRODUCTION}

Adhoc wireless network is a special case of wireless network devoid of predetermined backbone infrastructure. This feature of the wireless adhoc networks makes it flexible and quickly deployable. Nevertheless, significant technological challenges are also posed by this property. There are several challenges incorporating issues of efficient routing, medium access, power management, security and "Quality of Service" (QoS). As the nodes correspond over wireless links, all the nodes must combat against the extremely erratic character of wireless channels and intrusion from the additional transmitting nodes. These factors make it a challenging problem to exploit on data throughput even if the user-required QoS in wireless adhoc networks is achieved [1].

A source node that needs to communicate with a destination node uses either a direct link or a multihop route to reach the latter. This requires that all nodes must have some basic routing capability to ensure that packets are delivered to their respective destinations [2]. Repeated route changes cause huge complications in implementing adhoc networks owing to the mobility of the nodes and intrusion between nodes [1]. The high packet loss rates and recurrent topological changes lead to unbalanced transport layer and constrained amount of traffic being carried out by the network. The three eminent problems in adhoc networks are the lack of constant packet delivery due to the intrusion and movement of nodes, incomplete bandwidth owing to the channel limitations, and constrained node life span caused as an outcome of small battery size [1]. A major challenge in mobile adhoc networking is how to maximize data packet delivery in the face of rapidly changing network topology without incurring energy [2].

\section{RELATED WORKS}

Over the last few years, several routing protocols are proposed for mobile adhoc networks [3]-[9], [14], [15]. A number of performance comparison studies [10]-[13] have revealed that the on-demand routing protocols perform better in terms of packet delivery and routing overhead than proactive routing schemes especially in the presence of node mobility. Proactive and hybrid schemes do not perform well in dynamic topologies because of the following two major factors: Slow detection of broken links and periodic exchange of route updates even when routes are not needed [2].

\section{WORKING MECHANISM OF ANT COLONY OPTIMIZATION}

The basic idea of the ant colony optimization meta heuristic is taken from the food searching behavior of real ants. When ants are on they way to search for food, they start from their nest and walk toward the food and when an ant reaches an intersection, it has to decide which branch to take next. While walking, ants deposit pheromone, which marks the route taken. The concentration of pheromone on a certain path is an indication of its usage. With time the concentration of pheromone decreases due to diffusion effects. This property is important because it is integrating dynamic aspect into the path searching process [16].

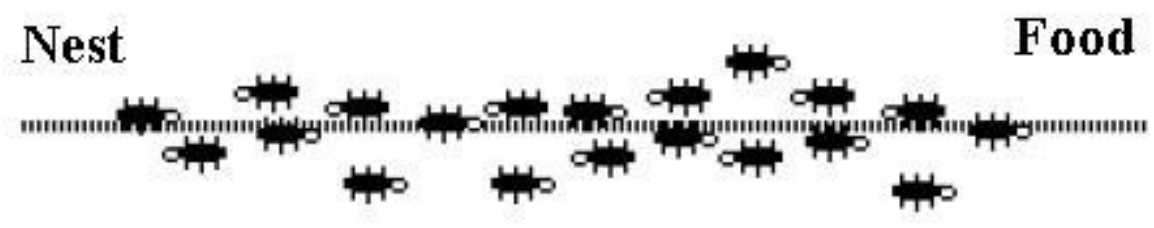

Figure 1: Ants travel from Nest to Food [16] 


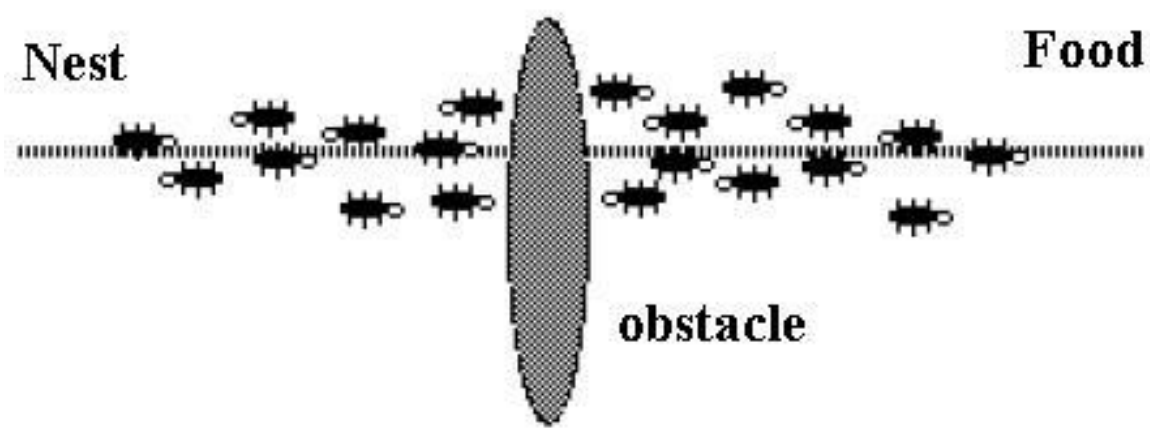

Figure 2: Ants find an obstacle [16]

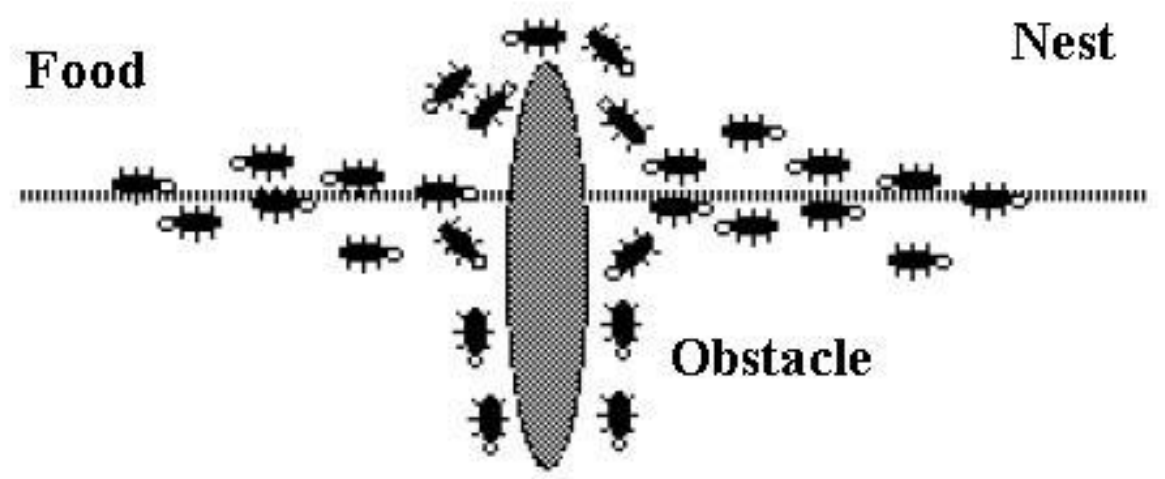

Figure 3: Ants try to find alternative route [16]

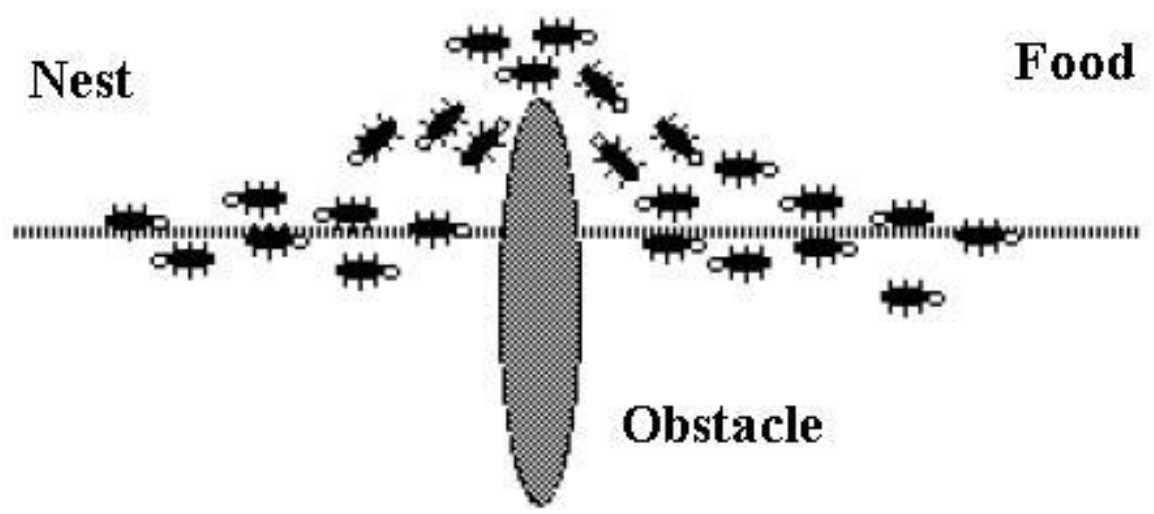

Figure 4: Ants travel through shortest route [16]

Fig. 1 to 4 show a scenario with a route from the nest to the place of food in which all ants follow the pheromone trail. Suddenly, an obstacle gets in their way so the first ants randomly select the next branch between the two branches: the upper and lower branches. Since the upper route is shorter than the lower one, the ants which take this shorter path will reach the place of food first. On their way back to the nest, the ants again have to select a path. After a short time the pheromone concentration on the shorter path will be higher than on the longer path, because the ants using the shorter path will increase the pheromone concentration faster. The shortest path will thus be identified and eventually all ants will only use this one. This behavior of the ants can be used to find the shortest path in networks [16].

Especially, the dynamic component of this method allows a high adaptation to changes in mobile adhoc network topology, since in these networks the existence of links are not guaranteed and link changes occur very often [17]. The simple ant colony optimization meta-heuristic shown in the previous section illustrates different reasons why this kind of algorithms could perform well in mobile multi-hop adhoc networks. We will discuss various reasons by considering important properties of mobile adhoc networks [17].

- ACO is based on agent systems and works with individual ants and This allows a high adaptation to the current dynamic topology of the network;

- ACO is based only on local information, that is, no routing tables or other information blocks have to be transmitted to neighbors or to all nodes of the network; 
- It is possible to integrate the connection/link quality into the computation of the pheromone concentration;

- Each node has a routing table with entries for all its neighbors, which contains also the pheromone concentration [16].

\section{DELAY AND ENERGY CONSCIOUS ROUTING PROTOCOL (DECRP)}

DECRP is an on-demand unipath routing protocol. The proposed DECRP takes advantage of various features of ant colony optimization and finds the delay and energy conscious path towards the destination node. As said in ACO, the pheromone is the key for the ants to find the best path from nest towards the food. In DECRP, the route request packets (RREQ) are considered to be the forwarding ants (FA). The FA will find the efficient node and establishes the pheromone (link). The pursuing ants (PA) are the data packets which follow the same path towards the destination. When there is any change in the network topology (obstacle) due to the mobility of the nodes, the FA using the efficient node selection mechanism and start communicating the packets through the efficient node.

\subsection{Estimating Delay}

It is important to estimate delay in mobile ad hoc networks for path optimization. Hence this research focuses to evaluate delay in an exact way. Each mobile node in the ad hoc network is considered as buffer. The data packets or RREQ packets arrive the buffer with poisson distribution and it is referred by $\lambda$ Hence the delay of the node can be computed by the following equation.

$$
\text { Delay }=\frac{\lambda T_{2}}{2(1-\sigma)}+T_{1}
$$

- $\lambda$ is the arriving rate of data packets to the buffer.

- $\quad \mathrm{T} 1$ is the mean service time required to transfer a data packet with success (which also includes retransmission delays).

- $\sigma$ is the rate occupation which is equal to $\lambda_{\mathrm{T} 1}$.

- $\mathrm{T} 2$ is the second moment of service time distribution.

\subsection{Estimating Residual Energy}

The information about the residual energy of the neighbor nodes is stored by every node throughout requesting the other nodes about their residual energies. The residual energies at a node can be calculated as Residual energy $=$ initial energy - consumed
energy ---- (2)

\subsection{Efficient Node Selection Mechanism}

The efficient node selection algorithm uses two distinct parameters and they are delay and residual energy. By estimating the two parameters the efficient node selection will be carried out using the below equation

node_cost $=$ energy $*(1 /$ delay $)$----- (3)

The node with the least cost value is selected and the pheromone (link) is established.

\section{SIMULATION MODEL AND PARAMETERS}

NS2 is used to simulate proposed protocol in our simulation; the channel capacity of mobile hosts is set to the same value: 2 Mbps. The distributed coordination function (DCF) of IEEE 802.11 for wireless LANs as the MAC layer protocol has been used. It has the functionality to notify the network layer about link breakage. In our simulation, 50 to 200 mobile nodes move in a 1500 meter x 1500 meter rectangular region for 100 seconds simulation time. Assume each node moves independently with the same average speed. All nodes have the same transmission range of 250 meters. In our simulation, the speed is set as $5 \mathrm{~m} / \mathrm{s}$. The simulated traffic is Constant Bit Rate (CBR).

The simulation settings and parameters are described in table 1 .

Table1. Simulation settings and parameters

\begin{tabular}{|l|l|}
\hline No. of Nodes & $50,75,100,125,150$ and 200 \\
\hline Area Size & $1500 \times 1500$ meters \\
\hline MAC & $802.11 \mathrm{~b}$ \\
\hline Radio Range & 250 meters \\
\hline Simulation Time & 100 seconds \\
\hline Traffic Source & CBR \\
\hline Packet Size & $512 \mathrm{~KB}$ \\
\hline Mobility Model & Random Waypoint Model \\
\hline Speed & $5 \mathrm{~m} / \mathrm{s}$ \\
\hline Initial Energy & 0.5 Joules \\
\hline
\end{tabular}

\section{Performance metrics}

Routing overhead, delivery ratio, delay and total energy consumption of nodes.

\section{RESULTS}

In fig. 5 the number of overhead packets is plotted in y-axis and $\mathrm{x}$-axis shows the number of nodes ranging from 50 to 200 . It can be clearly seen from fig. 5 that the overhead packets are reduced in DECRP than AODV. In fig. 6 delivery ratio is plotted in $y$-axis and $\mathrm{x}$-axis shows the number of nodes ranging from 50 to 200 and is observed that the delivery ratio of packets is increased in DECRP than AODV. In fig. 7 the delay in seconds are plotted in y-axis and the number of nodes ranging from 50 to 200 in $x$-axis and it is proved that the delay is reduced in DECRP than AODV. In fig. 8 the energy consumption of nodes which are measured in joules are plotted in $\mathrm{y}$-axis and $\mathrm{x}$-axis shows the number of nodes ranging from 50 to 200 . It can be observed from fig. 8 that the total energy consumption of nodes is reduced in proposed DECRP than AODV. 


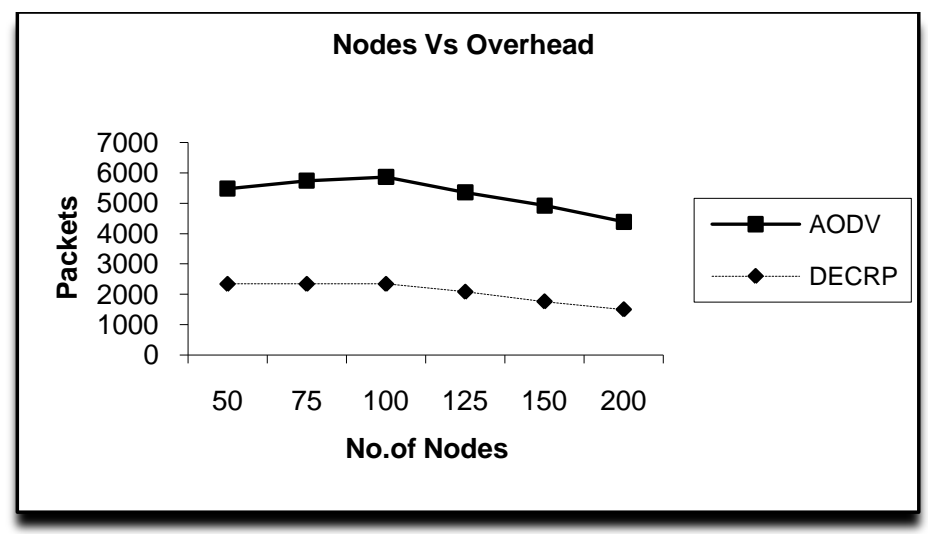

Figure 5: Number of Nodes Vs Overhead Packets

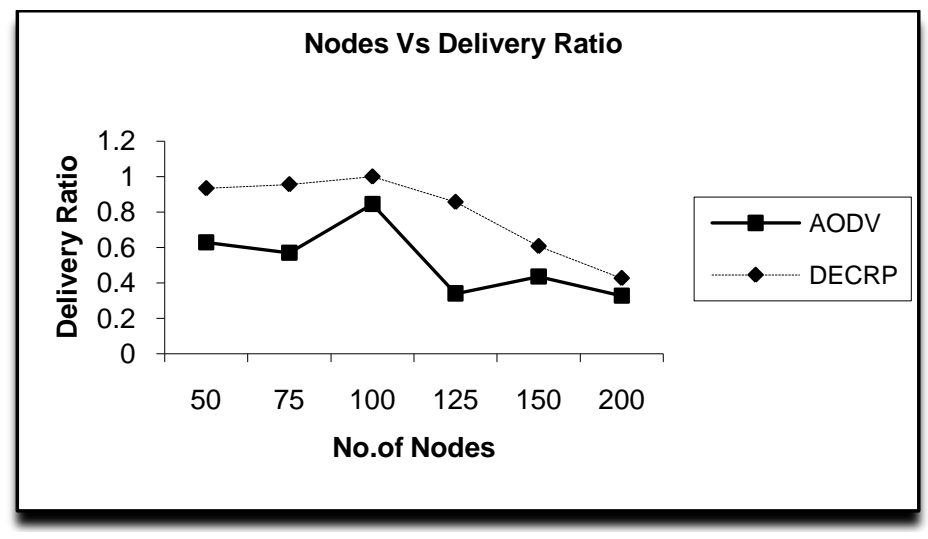

Figure 6: Number of Nodes Vs Packet Delivery Ratio

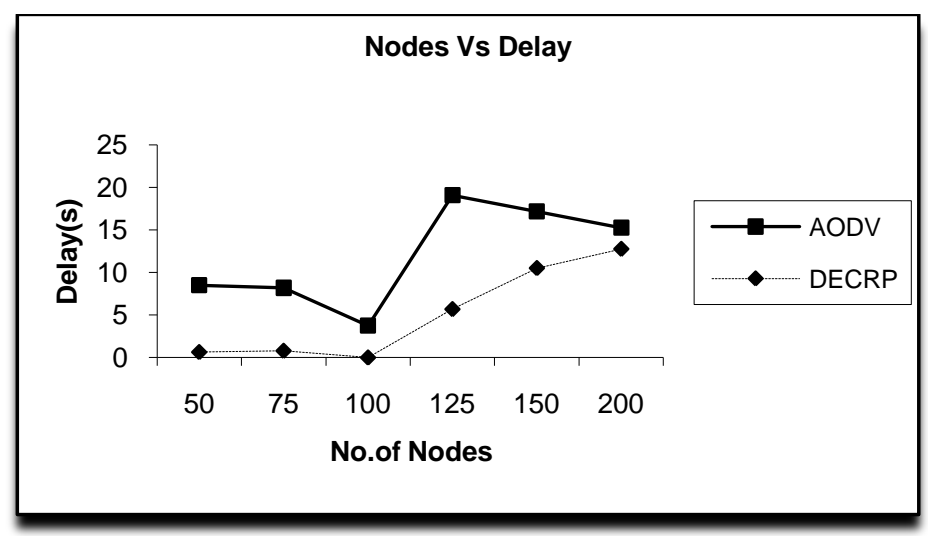

Figure 7: Number of Nodes Vs Delay (sec) 


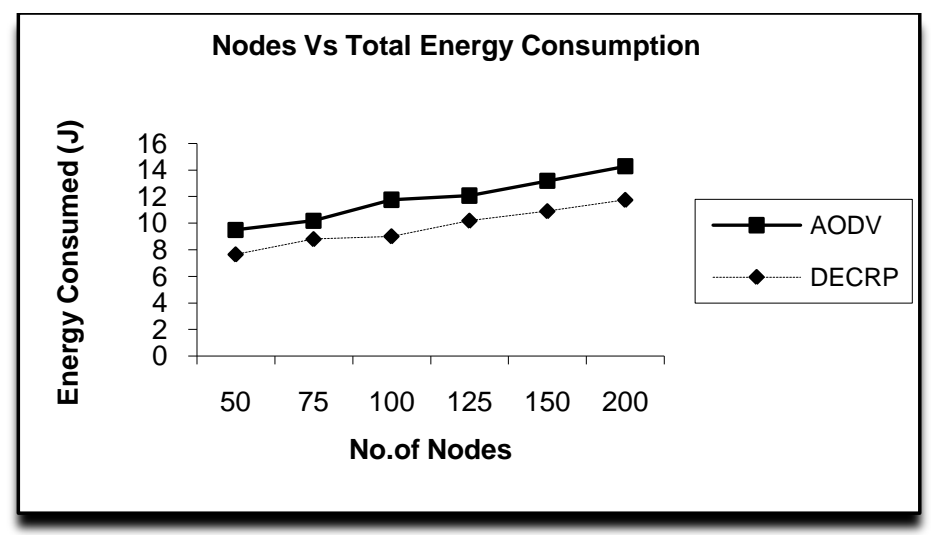

Figure 8: Number of Nodes Vs Total Energy Consumption of Nodes (J)

\section{CONCLUSION}

This paper presents a delay and energy conscious routing approach based on ant colony optimization. An adaptive and accurate delay estimation mechanism is proposed. Based on the estimated delay and residual energy of the nearby nodes, DECRP finds the efficient node and sends the data packets through that node.

\section{REFERENCES}

[1] S.Venkatasubramanian and Dr.N.P.Gopalan, "A QoSBased Robust Multipath Routing Protocol for Mobile Adhoc Networks", IACSIT International Journal of Engineering and Technology Vol.1,No.5, December, 2009.

[2] Alvin C. Valera, Student Member, IEEE, Winston K.G. Seah, Senior Member, IEEE, and S.V. Rao, Senior Member, IEEE, "Improving Protocol Robustness in Ad Hoc Networks through Cooperative Packet Caching and Shortest Multipath Routing", IEEE Transactions On Mobile Computing, Vol.4, No. 5, September/October 2005.

[3] Charles E.Perkins and Elizabeth M. Royer, "Ad-hoc OnDemand Distance Vector Routing", in proceedings of 2nd IEEE Workshop on Mobile Computing Systems and Applications, pp: 90- 100, 25- 29 Feb, New Orleans, LA, USA, 1999, Doi: 10.1109/MCSA.1999.749281.

[4] David B. Johnson and David A. Maltz, "Dynamic Source Routing in Ad Hoc Wireless Networks", in proceedings of International Series in Engineering and Computer Science, vol. 353, pp: 153- 181, 1996.

[5] Xiaobing Hou and David Tipper, "Impact of Failures on Routing in Mobile Ad Hoc Networks Using DSR", in proc. of IEEE Journal on Selected Areas in Communications, vol. 8, no. 9, pp. 1696-1708, Dec. 1990.

[6] Hossam Hassanein and Audrey Zhou, "Routing with Load Balancing in Wireless Ad hoc Networks", in proc. of 4th ACM International Workshop on Modeling, Analysis and Simulation of Wireless and Mobile Systems, pp: 89- 96, Rome, Italy, 2001.

[7] Ihab El Kabary, Amal Elnahas and Said Ghoniemy, "Weighted Critical Path Routing Protocol for Mobile Ad Hoc Networks", in proc. of International Conference on Wireless Information Networks and Systems, Portugal, 2006.

[8] Lei Chen and Wendi B. Heinzelman, "QoS-Aware Routing Based on Bandwidth Estimation for Mobile Ad Hoc Networks", in proceedings of IEEE Journal on Selected Areas in Communications, vol. 23, no. 3, March 2005.
[9] Kaixin Xu, Ken Tang, Rajive Bagrodia, Mario Gerla and Michael Bereschinsky, "Adaptive Bandwidth Management and QoS Provisioning in Large Scale Ad Hoc Networks", in proceedings of IEEE Conference on Military Communications, vol. 2, pp: 1018- 1023, 13- 16 October 2003, Doi: 10.1109/MILCOM.2003.1290305.

[10] Michael Gerharz, Christian de Waal and Matthias Frank and Paul James, "A Practical View on Quality-of-Service Support in Wireless Ad Hoc Networks", in Proc. of the 3rd IEEE Workshop on Applications and Services in Wireless Networks (ASWN), pp: 185-196, Berne, Switzerland, July 2003.

[11] Geetha Jayakumar and Gopinath Ganapathy, "Performance Comparison of Mobile Ad-hoc Network Routing Protocol", in proc. of International Journal on Computer Science and Network Security, vol. 7, no. 11, Nov. 2007.

[12] E. Ahvar and M. Fathy, "Performance Evaluation of Routing Protocols for High Density Ad Hoc Networks based on Energy Consumption by GlomoSim Simulator", in proceedings of World Academy on Science, Engineering and Technology, vol. 23, August 2007.

[13] Changling Liu and Jorg Kaiser, "A Survey of Mobile Ad Hoc network Routing Protocols", Technical Report, University of Magdeburg, October 2005

[14] Duc A. Tran and Harish Raghavendra, "Congestion Adaptive Routing in Mobile Ad Hoc Networks", IEEE Transactions on Parallel and Distributed Systems, vol. 17, no. 11, November 2006.

[15] Jianbo Xue, Patrick Stuedi and Gustavo Alonso, "ASAP: An Adaptive QoS Protocol for Mobile Ad Hoc Networks", 14th IEEE proceedings on Personal, Indoor and Mobile Radio Communications, vol. 3, pp: 2616- 2620, 7-10 September 2003, Doi: 10.1109/PIMRC. 2003.1259201.

[16] Ahmed M. Abd Elmoniem, Hosny M. Ibrahim, Marghny H. Mohamed, and Abdel-RahmanHedar, "Ant Colony and Load Balancing Optimizations for AODV Routing Protocol", International Journal of Sensor Networks and Data Communications, Vol. 1 (2012), Article ID X110203, 14 pages doi:10.4303/ijsnde/X110203.

[17] Mesut G"unes, Udo Sorges, Imed Bouazizi, "ARA - The Ant-Colony Based Routing algorithm for MANETs", InternationalWorkshop on Ad Hoc Networking (IWAHN 2002), Vancouver,British Columbia, Canada, August 18$21,2002$. 